\title{
Rosa roja - Rosa águila: simplemente Rosa Luxemburgo
}

\author{
Beatriz Rajland ${ }^{*}$
}

\section{Resumen}

Rosa Luxemburgo fue una revolucionaria integral, en producción teórica y en acción. Internacionalista, comunista de acción y pensamiento participativo, dedicada a defender los intereses de la clase obrera, preocupada por la libertad, la democracia y el desarrollo humano pleno y el antiburocratismo. Se distinguió por no aceptar fórmulas tradicionales, pero al mismo tiempo rigurosa consigo misma, no cesaba de reformular permanentemente sus ideas y las acciones que tuvieran a su entender un valor especial en la lucha política emancipadora. La "batalla de ideas" adquiere un lugar sumamente importante hoy en América Latina y el Caribe, como sustento básico en la articulación de lo social y lo político, para construir un futuro de emancipación, del que Rosa Luxemburgo fue una de las pioneras.

Palabras clave: Luxemburgo, Rosa, 1871-1919.

* Doutora em Direito da Universidade de Buenos Aires (UBA). Professora consultora da UBA. Vice-presidenta da Fundación de Investigaciones Sociales y Políticas (FISYP). 
Quiero hablar de Rosa Luxemburgo en el pensamiento universal, no como mujer revolucionaria, sino como revolucionaria universal.

¿Por qué aclaro esto? Porque lo contrario implica siempre y de alguna manera una discriminación de género: ¿su talla es la de una mujer revolucionaria o además de esa verdad objetiva, su pensamiento trasciende el género y se presenta como universalizado? Obviamente, es ambas cosas, pero frecuentemente sólo se toma la primera de las apreciaciones, su carácter de mujer revolucionaria, como si esa parte de la ecuación que sintetiza la propia Rosa Luxemburgo reemplazara la de su valor como revolucionaria universal en una suerte de primacía de lo particular por sobre lo general.

Sin embargo, no es tampoco posible dejar de considerar, en especial, el carácter de género respecto de esta revolucionaria que vivió en una época particularmente difícil para el desarrollo integral de la mujer. Pensemos por un momento en el costo social que podía significar desoír el "mandato social" que atribuía a la mujer, el ser mujer-madre, mujer-familia, mujer en los límites de la casa y no el ser partícipe de un mundo especialmente diseñado para el hombre, como era el de la política.

Rosa Luxemburgo fue una revolucionaria integral, en producción teórica y en acción. Pero Rosa Luxemburgo fue también una de las personalidades más controvertidas de la historia del movimiento socialista internacional. Sus detractores provienen de todas las tendencias, tanto de la clase dominante como de la izquierda tradicional. Se la ha distorsionado y calumniado, intentando presentarla como lo contrario de lo que fue: una revolucionaria.

Fue internacionalista, comunista de acción y pensamiento participativo, dedicada a defender los intereses de la clase obrera, preocupada por la libertad, la democracia y el desarrollo humano pleno y el antiburocratismo.

Aun considerando erróneos varios de sus posicionamientos sobre distintos aspectos de la teoría y la lucha, tanto Lenin como Trotsky la consideraron una revolucionaria cabal, representante destacada del proletariado revolucionario y del marxismo sin falsificaciones.

Así Lenin, en "Notas de un periodista" (LENIN, 1976), enumera una serie de análisis a su modo de ver equivocados que había formulado Rosa Luxemburgo, luego de lo cual dice:" [...] Pero a pesar de sus errores fue $-y$ para nosotros sigue siendo - un águila". Y responde a sus detractores con una frase de una vieja fábula rusa que dice: 
"A veces las águilas descienden y vuelan entre las aves de corral.

Pero las aves de corral jamás se remontarán hacia las nubes".

(Ivan Krylov, [ 18--?] )

Prevé que “[...] todo el mundo venerará su memoria y sus obras [...] [y que] serán útiles para la educación de muchas generaciones [...]” (LENIN, 1976, p. 255-256). Por su parte, Trotsky escribe un artículo en defensa de Rosa Luxemburgo que titula: "Fuera las manos de Rosa Luxemburgo" (TROTSKY, 1976), expresión más que elocuente para oponerse a las calumnias formuladas por Stalin. También Lenin (1976, p. 255) considera que hasta en los trabajos que, a su criterio, formula Rosa en forma equivocada "[...] se ven las alas del águila" [...]

Su gran amiga, la revolucionaria Clara Zetkin (2007, p. 109), la describe en estos términos": "[...] Severa con ella misma, tenía para sus amigos una indulgencia intuitiva...En relación a la amistad, ella era la encarnación misma de la fidelidad y de la devoción, de la abnegación y de una tierna solicitud [...]"

Y recuerda que Mehring “[...] afirmaba con razón que Rosa Luxemburgo era entre los discípulos de Marx la de más clara inteligencia. Perspicaz, profunda y totalmente independiente en su pensamiento [...]" (ZETKIN, 2007, p. 110).

En verdad, ella se distinguió por no aceptar fórmulas tradicionales, pero al mismo tiempo rigurosa consigo misma, no cesaba de reformular permanentemente sus ideas y las acciones que tuvieran a su entender un valor especial en la lucha política emancipadora.

La idea del socialismo era para Rosa Luxemburgo una pasión poderosa, absorbente, una pasión intelectual y moral. Esta pasión la encendía y se transformaba en obra creadora. Preparar la revolución que debía abrir la ruta al socialismo fue la tarea de toda su vida y el solo fin que conoció la ambición de esta mujer única "[...] ella ha sido el fuego de la Revolución [...]" (ZETKIN, 2007, p. 111). 


\section{Rosa Luxemburgo en el siglo XXI}

Quizás lo más actual de su pensamiento en el contexto de la globalización capitalista brutal y depredadora de hoy esté resumido en la síntesis con la que expresara su visión teórica y política de su mundo contemporáneo y de un mundo por venir: socialismo o barbarie.

Síntesis que, por otra parte, implica una continuidad, ya que recoge los análisis que Marx había formulado en igual sentido. Es dramática la alternativa formulada, pero es clara: o nos planteamos la construcción de socialismo, lo que implica ruptura respecto al sistema dominante, o el futuro de la sociedad estará condenado a la barbarie, en el sentido de agudización de las consecuencias de la explotación y el saqueo político, económico, social, cultural y ecológico capitalista. Basta con mirar a nuestro alrededor el mundo de hoy.

El capitalismo de esta época ha multiplicado sus rasgos de barbarie encarnados especialmente por la creciente militarización, la política de agresión y expansión imperialista, la destrucción progresiva del hábitat y el medio ambiente, la apropiación de los recursos naturales de los países subdesarrollados o periféricos o "tercermundistas", por parte del poder de las multinacionales y los países centrales, hegemónicos, de "primer mundo".

Los ejemplos están a la vista:

- las invasiones a Afganistán y a Irak, las amenazas a Irán y Siria, a Cuba y Venezuela, la política israelí para con Palestina (impulsada por los EE.UU.);

- las políticas implementadas con el neoliberalismo, que trajeron como consecuencia para los pueblos: hambre, miseria, desocupación, pobreza, extrema desigualdad, discriminación, migraciones, enfermedades endémicas, retroceso educativo;

- la agresión al ecosistema, cuyas muestras más evidentes y contundentes son: el cambio climático, la contaminación del aire y el agua, la deforestación del planeta, la modificación de las tierras fértiles de los países periféricos, con la implantación de nuevos monocultivos como la soja y los agrocombustibles. Todo ello, resultado de la política de las multinacionales. 
La misma estrategia de la llamada "guerra infinita", llevada a cabo por los Estados Unidos, particularmente después de los atentados a las "torres gemelas" de 2001, ha significado una estrategia del apoderamiento de recursos naturales - principalmente gas, petróleo y agua - lisa y llanamente. Ninguna ficción de integración de mercados o de intercambio, sino la pretensión de la consagración por la fuerza de las armas, de que este mundo será global pero de globalizadores y globalizados y hegemonizado por los EE.UU.

En América Latina y el Caribe, los efectos de la "barbarie" descripta se pueden observar en toda su plenitud. La aplicación de los mandatos de las multinacionales, significados en los estragos de la aplicación del consenso de Washington, han hambreado a nuestros pueblos, llevando a cabo verdaderos saqueos de nuestras riquezas, imponiendo la sojización del campo y empobreciendo nuestras tierras, violando derechos humanos económicos y sociales básicos, criminalizando las protestas sociales, imponiendo muros antiinmigratorios, acuerdos denominados de libre comercio, tanto bilaterales como multilaterales - Área de Libre Comercio de las Américas (ALCA), que en realidad son libres sólo para las potencias hegemónicas.

No obstante el poder y la fuerza agresiva que supone lo enunciado, esa política devastadora ha comenzado a ser nuevamente enfrentada por la acción de los pueblos. La resistencia extendida en la última década, particularmente en América Latina, motiva a pensar en nuevos escenarios para la lucha de clases a escala global. Frente a un poder económico y político mundial y políticas globales sustentadas por los Estados nacionales y los organismos multilaterales, también asoma un bloque "alternativo". Son procesos (salvo en lo que refiere a Cuba) aun no definidos, pero que, en algunos casos, indican tendencias que avanzan en su articulación. Y eso es lo que se necesita. No son tampoco homogéneos, pueden advertirse fundamentalmente dos grupos: por un lado, Venezuela, Bolivia, Ecuador (con definiciones acerca de la necesidad de construir otra sociedad: socialismo del siglo XXI, socialismo comunitario), y por el otro, Nicaragua, Brasil, Argentina y Uruguay, que no cuestionan el sistema capitalista y también heterogéneos entre sí. En todos ellos, lo más importante es el registro de los cambios en la subjetividad de los pueblos.

Se están caminando ya algunos pasos con el avance de las propuestas del Alternativa Bolivariana para las Américas (ALBA), del Banco del Sur, del SUCRE. Las últimas reuniones, en ese sentido, de Cuba, Venezuela, 
Nicaragua y Bolivia abren la esperanza a nuevas formas de integración, en la que participen las organizaciones sociales.

Se está comenzando a popularizar la consigna del socialismo del siglo XXI, lanzada por el presidente Chávez, y no ya como mera consigna, sino como posible escenario a construir. Es volver a rescatar para el imaginario social que el capitalismo no construye soluciones para los sectores populares, sino que los sepulta cada vez más, y que hay una alternativa que llamamos socialismo, sobre el cual no sabemos aun demasiado, pero que tendrá futuro, siempre y cuando sea resultado de la movilización, acción y participación del movimiento popular, social y político. Es correr el velo, la ficción de esta sociedad hegemónica de explotación y barbarie.

Pero también, enlazando lo anterior e imaginando el significado de la acción hacia el socialismo, resulta actual la formulación de Rosa Luxemburgo acerca de reforma o revolución, o mejor expresado en el contexto contemporáneo, reforma y revolución.

Revolución significa ruptura, ¿anula entonces las posibilidades de realizar reformas en el seno del propio sistema capitalista? No se trata de eso, los programas de reformas son útiles en tanto y cuanto se tenga claro que el horizonte es la ruptura revolucionaria, porque si no, no hay ruptura.

Rosa Luxemburgo "[...] rechazó del reformismo, tanto económico como político, la ilusión de reformar el capitalismo, preservando de él lo bueno y desechando lo malo" (VALDEZ PAZ, 2001, p. 59). En cambio de lo que se trata es de vincular ambos extremos de la ecuación traducidos en objetivos inmediatos y objetivo final. Los inmediatos hacen parte de la táctica, y el final, de la estrategia, teniendo en cuenta también que la táctica no tiene autonomía de la estrategia y en este sentido la integra.

La participación activa y militante en sindicatos, partidos, movimientos sociales de amplio espectro como los que existen en la actualidad se inscribe en la táctica. Son herramientas políticas, importantes para la construcción de alternativa, para el proceso de construcción de poder contrahegemónico, pero insuficientes para la ruptura definitiva, sino se da el salto cualitativo: la conquista del poder político y/para la emancipación de los trabajadores. Esto quiere decir que se rechaza cualquier idea de que se arribe a ese salto cualitativo que es el socialismo por imperio del desarrollo de medidas como resultado de una evolución dada al interior del propio sistema. Dentro 
del propio sistema dominante, en definitiva lo que ocurre es su propia reproducción, aunque se logren establecer incluso algunos cambios por imperio de la movilización popular.

Así lo planteaba Rosa Luxemburgo de acuerdo a la introducción a su pensamiento que formula Waters (1976, p .48):

[...] ¿Es posible que la socialdemocracia [no en el sentido de socialdemocracia actual, sino de organización de los comunistas - BR] se oponga a las reformas? ¿Podemos contraponer la revolución social, la transformación del orden imperante, nuestro objetivo final, a la reforma social? De ninguna manera...Entre la reforma social y la revolución existe... un vínculo indisoluble. La lucha por reformas es el medio; la revolución social, el fin.

Decíamos de la actualidad de estos pensamientos, porque ya hace un tiempo que se ha producido una suerte de "reciclamiento" de la socialdemocracia (la de ahora, la posterior al debate crucial sobre el presupuesto de guerra - la 1 ra. Guerra - en el que tuvo crucial participación nuestra autora en comentario) que no cambia su carácter reformista. Nos estamos refiriendo a las ideas de "tercera vía", formuladas académicamente por el sociólogo Anthony Giddens y políticamente por el entonces Primer Ministro inglés, Tony Blair, que han intentado encubrir el giro a la derecha de una socialdemocracia que en Europa desarrolló las políticas neoliberales.

Pero también nos referimos a las ideas "posibilistas" (del mismo cuño de las de "tercera vía”) que se instalaron en Latinoamérica en muchos núcleos intelectuales y políticos, que "postergaron" el debate sobre los cambios profundos, revolucionarios, por el ejercicio de lo que se considera "posible".

Estos posicionamientos también confunden y manipulan.

Por ello, la "batalla de ideas" adquiere un lugar sumamente importante hoy en América Latina y el Caribe, se trata de confrontar, pero no diletantemente, sino hacia la acción. Este debería ser el sustento básico en la articulación de lo social y lo político, si queremos construir un futuro de emancipación, del que Rosa Luxemburgo fue una de las pioneras, proceso que ha de ser para poder ser: democrático y antiburocrático. 


\section{Nota}

1 Me voy a permitir citar la opinión de Zetkin con cierta extensión, ya que es invalorable para apreciar la talla de Rosa Luxemburgo y no siempre muy conocida en los aspectos que relata.

\section{Referencias}

LENIN, V. Apéndice. In: LUXEMBURGO, Rosa. In: Obras escogidas. Buenos Aires: Pluma, 1976. Tomo 2.

TROTSKY, L. Apéndice. In: LUXEMBURGO, Rosa : In: Obras escogidas. Buenos Aires: Pluma, 1976. Tomo 2.

ZETKIN C. Rosa Luxemburg: en Dans l'asile de nuit. Paris: Ediciones L'Herne, 2007.

VALDEZ PAZ, Juan, Rosa Luxemburgo, actualidad de su teoría política. In: Rosa Luxemburgo. Una rosa roja para el siglo XXI. Publicación del Encuentro Internacional Rosa Luxemburgo y los problemas contemporáneos, realizado en La Habana, Cuba por el Centro de Investigación y Desarrollo de la cultura Cubana Juan Marinello. Edición propia, año 2001.

WATERS, Mary-Alice, Introducción. In: LUXEMBURGO, Rosa.In:

Obras escogidas. Buenos Aires: Pluma, 1976. 


\section{Rosa vermelha - Rosa águia: simplesmente Rosa Luxemburgo}

\section{Resumo}

Rosa Luxemburgo foi uma revolucionária integral em sua produção teórica e na ação. Internacionalista, foi comunista na açáo e de pensamento participativo, se dedicou a defender os interesses da classe trabalhadora, preocupada pela liberdade, a democracia, o desenvolvimento pleno e o antiburocratismo. Distinguiu-se por não aceitar fórmulas tradicionais, mas, ao mesmo tempo, foi muito rigorosa consigo mesma, não cessando de reformular suas ideias permanentemente e as açốes que, no seu entendimento, tinham um valor especial na luta política emancipadora. A "batalha de ideias" adquire, hoje, um lugar muito importante na América Latina e no Caribe, como sustentaçáo básica na articulação do social e do político, para construir um futuro de emancipação do qual, Rosa Luxemburgo foi uma das pioneiras.

Palavras-chave: Luxemburgo, Rosa, 1871-1919.

\section{Beatriz Rajland}

E-mail: brajland@derecho.uba.ar

\section{Rosa red, Rosa eagle: simply Rosa Luxemburgo}

\section{Abstract}

Rosa Luxemburgo was a complete revolutionary in her theoretical production and action. An internationalist, she was a communist in action and in participatory thinking, who was dedicated to defending working class interests, with a constant concerned for liberty, democracy, complete development and for fighting bureaucracy. She was distinguished for not accepting traditional formulas, while remaining very rigorous with herself, never ceasing to reformulate her ideas and actions, which in her understanding, had a special value in a liberating political struggle. Today, the ?battle of ideas? acquires a very important place in Latin America and the Caribbean, as basic support in the articulation of the social and political realms, and the construction of a future of emancipation, of which Rosa Luxemburgo was a pioneer.

Key words: Luxemburgo, Rosa, 18711919. 